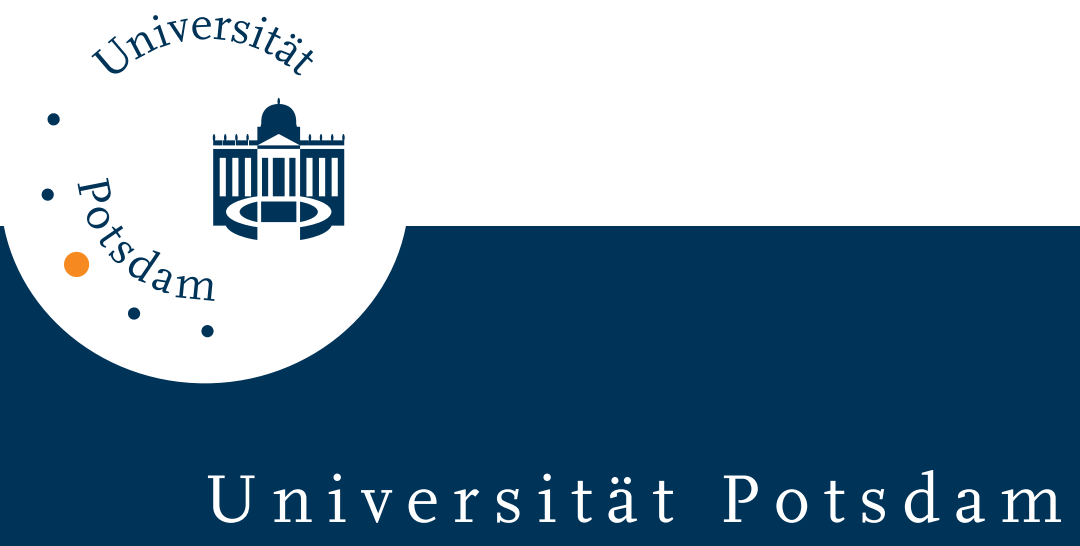

Diether Hopf

\title{
Mehrsprachigkeit und Schulerfolg bei Schülern mit Migrationshintergrund
}

first published in:

Sprache, Stimme, Gehör : Zeitschrift für Kommunikationsstörungen, 31 (2007) 4, ISSN 0342-0477

Postprint published at the Institutional Repository of the Potsdam University:

In: Postprints der Universität Potsdam

Humanwissenschaftliche Reihe ; 123

http://opus.kobv.de/ubp/volltexte/2009/3832/

http://nbn-resolving.de/urn:nbn:de:kobv:517-opus-38326

Postprints der Universität Potsdam

Humanwissenschaftliche Reihe ; 123 


\title{
Mehrsprachigkeit und Schulerfolg bei Schülern mit Migrationshintergrund
}

\author{
Bilingualism and School Achievement among Children of Migrant Background
}

Autor

Institut

\section{Hopf}

Max-Planck-Institut für Bildungsforschung, Berlin

\author{
Schl̈üsselwwörter \\ Zweisprachigkeit \\ Bilingualismus \\ Mehrsprachigkeit \\ - Herkunftssprache \\ - Verkehrssprache \\ - Lingua franca \\ - Schulleistungen \\ Migration \\ - Forschungsmethode \\ - Deutsch als Zweitsprache \\ Key words \\ - bilingualism \\ multilingualism \\ - family language \\ - lingua franca \\ school achievement \\ migration \\ - research methods \\ - German as a second \\ language
}

Bebliografie

DOI $10.1055 / \mathrm{s}-2007-985832$

Sprache · Stimme $\cdot$ Gehör

2007; 31: 163-169

(C) Georg Thieme Verlag KG

Stuttgart - New York

ISSN 0342-0477

Korrespondenzadresse

Prof. Dr. D. Hopf

Lützelsteiner Weg 45

14195 Berlin

dhopf@uni-potsdam.de

\section{Żusamumentassung \\ $\nabla$}

Nach wie vor wird kontrovers diskutiert, wie Schule und Unterricht mit den Herkunftssprachen der Schüler mit Migrationshintergrund umgehen sollten und welche Rolle der Verkehrssprache Deutsch zukommt. Wird von den einen die besondere Bedeutung der Herkunfts- oder Familiensprachen für die Entwicklung der Verkehrssprache und der Schulleistungen betont, schreiben andere vor allem der Verkehrssprache eine Schlüsselfunktion für das Erreichen guter Lernergebnisse in der Schule zu. Nach einem Überblick über die quantitativen Rahmenbedingungen, unter denen Schüler mit Migrationshintergrund und ihre Lehrer arbeiten, und nach einem Überblick über die sprachliche Differenzierung, die an den deutschen Schulen anzutreffen ist, werden zunächst die Erwartungen skizziert, die an den Gebrauch von Herkunftssprachen bzw. des Deutschen in der öffentlichen Diskussion geknüpft werden. Im Anschluss daran wird der Stand der Forschung über den Zusammenhang von Familiensprache und Verkehrssprache sowie von Sprache und Schulleistungen bei den Schülern mit Migrationshintergrund skizziert und methodenkritisch diskutiert. Aufgrund der lückenhaften Forschungsbefunde werden schließlich pädagogisch-psychologische Untersuchungen zum schulischen Lernen auf ihre Relevanz für die anstehende Frage geprüft. Schlussfolgerungen für die schulische Praxis, die dem Stand unseres Wissens entsprechen, sowie Aufgaben für künftige Forschung werden abschließend formuliert.

\section{Abstract \\ T}

As yet, no consensual answer has been given to the question of how the native languages of students of migrant background and the lingua franca (German) should be dealt with by the school and in the classroom. The article critically evaluates pertinent research, focusing on the research methods applied, and reaches the conclusion that the competence in native languages apparently does not foster the learning of the lingua franca. As far as school achievement is concerned, competence in the lingua franca seems to be very closely connected with school success. Recently published studies which contain evidence of causal relationships between language competence and school achievement are especially closely examined. Furthermore, an intervention study comparing types of treatments for language learning is discussed and the relevance of broader studies in psychological learning theory to school learning is inquired. Finally the consequences of the findings for school practice and future research are summarized and discussed:

- About one third of all students in school age are and will be children of migrant background; their presence will be a constant task of the German schools.

- These students come from more than $\mathbf{1 5 0}$ countries and speak close to 200 family languages; the need for a lingua franca is evident.

- Until today their school achievement is much worse than that of students without migrant background, the difference being between 2 and 3 school years.

- There is a strong co-variation between school achievement and competence in the German language which leads to the recommendation to invest into intense training of the language of the school, without recurrence to the family languages. 
- Gifted students with migrant background show good school achievements even if they operate in both languages, their family languages and the lingua franca; treatments in school should become more differentiated.

- Students without any knowledge of German also have to be differentially treated in the classroom.

The important role of the family languages for the individual and for society has to be put into the focus of public discussion.

- Future research should be directed towards finding causal relationships between teaching and learning outcomes and compare different treatments with German as a second language.

\section{Lermziel}

\%

Kenntnisse über Beziehungen zwischen schulischem Lernerfolg und Sprachkompetenz bei Migrantenkindern.

\section{Eimleitumg \\ .}

Im folgenden Beitrag geht es um den aktuellen Stand unseres Wissens über das Verhältnis von Zwei- oder Mehrsprachigkeit zu den Schulleistungen bei Schülern mit Migrationshintergrund Eng verbunden damit ist die Frage nach dem Verhältnis der Herkunfts- (oder Familien-)sprachen zur Verkehrssprache, dem Deutschen. Es gibt über diese Thematik international eine fast unübersehbare Forschungsliteratur, wovon hier vor allem die neu erschienenen Arbeiten thematisiert werden sollen. Diese spiegeln interessante Entwicklungen wider und geben Hinweise für den Umgang mit Zweisprachigkeit in der Schulpraxis.

\section{Quantitative Rahmendaten \\ $\nabla$}

Zunächst ist ein kurzer Überblick über den Umfang und die Zusammensetzung der hier interessierenden Schülergruppe notwendig, weil nur so die Aktualität und Brisanz des Themas deutlich werden kann.

Eine große und immer noch steigende Anzahl (und eine steigende Quote!) der Schüler in unseren öffentlichen Schulen hat einen Migrationshintergrund. Diese Kinder wachsen überwiegend mit einer anderen Erstsprache als Deutsch auf.

Brauchbare Daten für unsere Frage kommen weniger aus den offiziellen Statistiken, in denen z. B. die Aussiedler wegen ihres deutschen Passes nicht getrennt ausgewiesen sind, sondern aus repräsentativen sozialwissenschaftlichen Studien; die neuesten stammen aus der zweiten PISA- Studie [1], in der die einbezogenen Schüler im Alter von 15 Jahren Auskunft gegeben haben u. a. über die Herkunft ihrer Eltern und ihren Sprachgebrauch zu Hause und im Alltag.

Wir können danach folgende Angaben machen:

In Gesamt-Deutschland hat ein gutes Fünftel aller 15-jährigen Schüler einen Migrationshintergrund. Die Quoten in den Bundesländern sind sehr verschieden und betragen bis über ein Drittel der Schülerschaft. Die höchsten Quoten finden wir in Bremen (36\%), Hamburg, Baden-Württemberg und Hessen, die niedrigsten in Thüringen (4\%), Sachsen-Anhalt und Mecklenburg-Vorpommern. Bayern liegt mit 21\% im Durchschnitt der BRD.
Ein Drittel bis ein Fünftel der Schüler in der Mittelstufe der westdeutschen Sekundarschulen sind also Migrantenschüler. In niedrigeren Klassenstufen, z.B. in der Grundschule, sind die Quoten noch höher; die Tendenz ist steigend. Die Schüler leben in Deutschland nicht nur in Ballungszentren, sondern - das ist wichtig - zu großen Teilen auch in kleineren Städten oder auf dem Land.

Betrachtet man die Herkunft dieser Schüler, so rekrutieren sie sich aus über 150 Ländern. Die Mehrzahl von ihnen (ca. 70\%) gehört derzeit drei großen Gruppen an. Dies sind die Kinder:

- aus der Türkei

- aus der ehemaligen Sowjetunion

- die zu einer Sammelgruppe von Nationen gehörigen Schüler, die jeweils nur durch eine kleine Anzahl von Personen vertreten ist. In dieser Sammelgruppe stecken über 100 verschiedene Herkunftsländer und noch mehr Familiensprachen.

Von den drei großen Gruppen bilden die Türken mit 19\% die kleinste. Die zweitgrößte Gruppe sind die Schüler aus der ehemaligen Sowjetunion mit $24 \%$. Die größte Gruppe von allen aber ist die Sammelgruppe: über 26\% der Migrantenschüler stammen aus einer dreistelligen Zahl von Ländern. Dieser Tatbestand ist für unsere Frage von Bedeutung.

Es ist wichtig, sich diese Vielfalt klar zu machen angesichts der irreführenden Tendenz in der öffentlichen Diskussion, meist automatisch an türkische Schüler zu denken, wenn von Migrantenschülern die Rede ist.

\section{Rahmendaten zum Sprachgebrauch}

$\checkmark$

Die bei uns lebenden Migrantenschüler kommen nicht nur aus einer großen Zahl von Ländern, sondern sprechen eine noch größere Zahl verschiedener Sprachen - die Zahl liegt nahe bei 200; allein für Westberlin waren z.B. Ende der 80er Jahre 187 verschiedene Familiensprachen in der offiziellen Schulstatistik ausgewiesen. Für die Beschulung ist dabei von Bedeutung, dass es in Deutschland nur eine geringe Ghettobildung gibt, sodass im Regelfall in einer Schulklasse Schüler aus unterschiedlichen Herkunftsländern sitzen. Wir haben also in Deutschland nicht die komfortable Situation, die man z. B. in den USA vorfindet, wo die ganz überwiegende Mehrzahl der anderssprachigen Kinder Spanisch spricht. In unseren Schulen herrscht vielmehr eine multilinguale, keine bilinguale Situation vor. In Deutschland gibt es deshalb einen besonders großen Bedarf nach einer Lingua franca, einer gemeinsamen Sprache für alle.

Wie benutzen die Migrantenschüler ihre Sprachen? Genauer: welche Sprache verwenden sie in Schule und Freizeit, z.B. beim 
Kopfrechnen; beim Lesen und Schreiben von Briefen und EMails, beim Betrachten von Videos und Fernsehen? Darüber wissen wir seit der Veröffentlichung der erwähnten zweiten PISA-Studie erstmals Genaueres, jedenfalls was die 15-jährigen Schüler (meist Klasse 9) betrifft; übrigens auch Genaueres als in vielen anderen Ländern bekannt ist.

In dieser Studie wurden die Migrantenschüler sowohl nach dem Gebrauch der Herkunfts- oder Familiensprache (L1) als auch der Verkehrssprache Deutsch (L2) befragt. Auf der Grundlage der Ergebnisse kann man die Schüler in 3 Gruppen einteilen [2]:

deutschsprachige (sprechen im Alltag öfter Deutsch als die L1)

- zwei-oder mehrsprachige (sprechen Deutsch und die andere(n) Sprache(n) in gleichem Umfang)

- fremdsprachige (sprechen die L1 häufiger als Deutsch)

Die Verteilung in der BRD (über alle Nationen) sieht folgendermaßen aus: 51\% der Migrantenschüler sind deutschsprachig; $31 \%$ sind zwei- oder mehrsprachig; $13 \%$ fremdsprachig.

Dabei weisen die Schüler je nach Herkunftsland große Unterschiede im Sprachgebrauch auf. Beispielsweise sind bei den Türken nur 32\% deutschsprachig, bei den Polen hingegen $64 \%$. Und unter den „Fremdsprachigen“ findet man das Maximum von $20 \%$ bei den Türken und den Schülern aus der ehemaligen Sowjetunion. Die besten Werte (im Sinne einer Beteiligungsmöglichkeit am deutschsprachigen Unterricht) - hohe Deutschsprachigkeit bzw. niedrige Fremdsprachigkeit - weisen Polen und die Angehörigen der Sammelgruppe aus vielen verschiedenen Herkunftsstaaten auf.

Es gibt nun zwei große Fragen in unserem Zusammenhang, die im Übrigen nicht nur im schulischen Kontext von Bedeutung sind:

Wie steht es um das Verhältnis von L1 und L2 zueinander? Entwickeln sich die sprachlichen Kompetenzen unabhängig voneinander? Fördert das Erlernen der L1 das Lernen der L2, oder ist es eher hinderlich?

Wie hängen Zwei- (oder Mehr-)sprachigkeit und Schulleistungen zusammen?

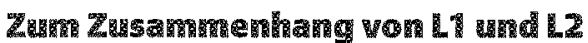

F

Von früh an, seit die „Gastarbeiterkinder" in deutschen Schulen als besondere Gruppe wahrgenommen worden sind, hat man der Muttersprachenfrage besondere Aufmerksamkeit gewidmet.

Die folgenreichste Annahme hinsichtlich der Bedeutung des Erlernens der L1 für den Erwerb der L2 bei Migrantenschülern und für ihre geistige Entwicklung, die auch heute noch virulent ist und sowohl in wissenschaftlichen Publikationen wie in den Medien immer wieder vorgetragen wird, ist die von Cummins. Cummins hat 1979 [3] (1984 auf deutsch) eine Hypothese formuliert, wonach ein Kind in „doppelter Halbsprachigkeit“ endet, wenn es nicht zunächst seine Erstsprache ausentwickelt hat bis etwa zum Stadium der formalen Operationen sensu Piaget (also bis etwa zum 10. oder 11. Lebensjahr). Cummins selbst hat das übrigens als Hypothese formuliert, weil es keine tragfähigen Belege aus der Forschung dafür gab. Seine Hypothese ist aber in Deutschland sofort wie ein fester Befund rezipiert worden und wird auch heute noch verwendet als Argument für bilinguale Erziehung von früh an, für muttersprachlichen Unterricht in verschiedenen Varianten etc., eben weil man glaubt, dass das Erlernen der L1 von größter Bedeutung sei für das Erlernen der
L2 und außerdem für die geistige Entwicklung insgesamt, und somit auch für die Schulleistungen.

Wenn man überprüft, ob sich die Cummin'sche Hypothese in der bisherigen Forschung bestätigt findet, so zeigt sich, kurz gesagt: es gibt bis heute keinen tragfähigen Beleg für ihre Richtigkeit. Kinder und Jugendliche brauchen keine vorab ausentwickelte L1 für das optimale Erlernen der L2; sie müssen auch nicht die L1 beherrschen, um begabungsgerechte Schulleistungen zu erzielen. Jedenfalls lässt sich die erwartete Wirkungsrichtung empirisch nicht finden [4].

Cummins ist nicht der einzige Wissenschaftler, der über den Zusammenhang von L1 und L2 geforscht hat, wenngleich er die bei weitem stärkste Wirkung in der öffentlichen Diskussion entfaltet hat. Im Folgenden sollen weitere Untersuchungen zu diesem Thema im Überblick dargestellt werden.

Zunächst aber eine wichtige Vorbemerkung:

Es geht bei den folgenden Ausführungen ausdrücklich nicht um die kleine Gruppe der genuin zwei- oder mehrsprachigen Personen, von denen wir alle persönlich den einen oder anderen kennen und bewundern, oder um solche Sprachkönner wie Martin Buber oder Jorgos Papandreou oder Felix Pollak, die ganz offensichtlich in zwei oder mehr Sprachen vollkommen zu Hause sind. Solche Menschen gehören meist zu den Hochbegabten, denen keine wie auch immer geartete Schule oder Sprachenfolge etwas anhaben kann.

Sondern es geht hier um alle Schüler, und das heißt, mehrheitlich um die große Zahl der ganz normalen, durchschnittlich interessierten und durchschnittlich lernfähigen Kinder; sowie auch um die lernlangsamen Kinder. Sie machen das Gros der Schülerschaft aus, ihnen muss sich Schule und Unterricht widmen, muss die richtigen Akzente für ihre Förderung setzen und Überforderung vermeiden. Letzteres ist besonders wichtig im Falle der Schüler mit Migrationshintergrund, weil diese in jeder Hinsicht ungleich umfangreichere Lernaufgaben zu bewältigen haben als Schüler ohne Migrationshintergrund; gerade auch jenseits von Schule und Unterricht.

Die empirischen Untersuchungen über Auswirkungen bilingualer Sprachförderung auf den Erwerb der Verkehrssprache, die unabhängig von der Theorie von Cummins durchgeführt wurden, weisen, was die methodische Anlage betrifft, eine große Vielfalt auf: es gibt kleine Fallstudien (zahlreich); experimentelle Untersuchungen (sehr selten); Surveys; Großuntersuchungen mit sechsstelligen Schülerzahlen.

Erfreulicherweise kommen schon seit Jahren so genannte Metaanalysen der Originalstudien auf den Markt, das sind Übersichten über die Forschungsliteratur, bei denen die Untersuchungen nach klar definierten methodischen Kriterien ausgewählt und miteinander verglichen werden. Diese Metaanalysen kamen allerdings zu widersprüchlichen Endergebnissen: Während die einen fanden, dass Unterricht in der L1 die L2 fördert, ergab sich bei anderen, dass bilinguale Beschulung Nachteile mit sich bringt.

$\mathrm{Zu}$ erklären ist diese Diskrepanz vor allem mit den unterschiedlichen methodischen Instrumenten, welche zu den verschiedenen Zeitpunkten zur Verfügung standen oder welche den jeweiligen Autoren geläufig waren, so dass manche Original-Untersuchungen für akzeptabel gehalten wurden, die dies nach neueren - oder nach anspruchsvolleren - methodischen Standards nicht sind. Insofern ist es ein Glücksfall, dass diese Arbeiten kürzlich erneut gründlich und nach besonders strengen forschungsmethodischen Kriterien durchgesehen worden sind; 
zuletzt vor ein paar Monaten von Limbird und Stanat vom MaxPlanck-Institut für Bildungsforschung [5].

Was ist nach dieser Übersicht der heutige Stand unseres Wissens?

Die Befunde aus den zahllosen Einzelstudien, aber auch aus den überblicksartigen Metaanalysen, bleiben - auch nach heutigem Stand - widersprüchlich. Man kann derzeit keine sicheren Schlüsse ziehen, weder zugunsten noch zu Ungunsten bilingualer schulischer Angebote. Weder gibt es belastbare Nachweise darüber, ob und in welchem Ausmaß die schulische Förderung der L1 den Erwerb der L2 begünstigt, noch ob sie ihn behindert. Die widersprüchliche Befundlage hat ihren Grund vor allem in einigen methodischen Schwächen vieler Untersuchungen zum Verhältnis der L1 zur L2, von denen einige genannt werden sollen:

- Bei denjenigen Studien, die Vorteile für bilinguale Programme in der Schule gefunden haben, muss man meist konstatieren, dass die Kontrollgruppen einem relativ unqualifizierten Unterricht ausgesetzt waren, während die bilingualen Experimentalgruppen erstklassigen Unterricht bei den Experimentallehrern erhielten. Vor allem: Es gibt keine Studie, bei der die im bilingualen Unterricht übliche zusätzliche Lernzeit in der L1 zu einer entsprechenden langen qualifizierten Förderung der L2 bei den Kontrollgruppen in Konkurrenz getreten wäre.

- Zum Forschungsstand ist außerdem zu vermerken, dass praktisch keine brauchbaren Untersuchungen vorliegen, die Auskunft geben über die Auswirkungen unterschiedlicher monolingualer Ansätze (z.B. Deutsch als Zweitsprache; oder Konzentration auf die Schulsprache).

- Der Mangel an empirischer Forschung in der BRD ist im Übrigen besonders bedauerlich, da der Föderalismus für die Forschung eigentlich eine günstige Situation schafft, indem er Vergleiche zwischen unterschiedlichen Ansätzen ermöglicht. Wir haben z.B. weder Studien darüber, ob das so genannte Bayerische Modell (ein bilinguales Transitionsmodell) sich günstiger auf die schulische und die sprachliche Entwicklung der Schüler mit Migrationshintergrund ausgewirkt hat als z.B. das Nordrhein-Westfälische Modell mit seinen Vorbereitungsklassen oder das Berliner Modell mit seinem Konsulatsunterricht oder die bundesweit in vielen Schulen vorherrschende Einbeziehung der Migranten in den Regelunterricht ohne jede zusätzliche Förderung bzw. mit DaZ (Deutsch als Zweitsprache). Um so heftiger waren die öffentlichen Kontroversen über die vermeintlichen Vorzüge oder Nachteile der Modelle.

\section{Zum Zusammenhang von Zweisprachiglkeäc und Schalleistung}

Deutlich besser sieht die Forschungslage zum Thema Zweisprachigkeit und Schulleistung aus. Seit mehr als 35 Jahren ist bekannt - und dieser Befund wiederholt sich bis heute -, dass die Schüler mit Migrationshintergrund erheblich niedrigere Schulleistungen aufweisen als die Schüler ohne Migrationshintergrund; wobei die Situation in Deutschland im internationalen Vergleich besonders negativ auffällt. Die frühen Untersuchungen mussten allerdings ohne repräsentative Testergebnisse auskommen und haben die Leistungsdifferenzen nur anhand der unterschiedlichen Quoten von Migrantenkindern in den Sekundarschultypen dokumentieren können. So ist auch heute noch der relative Schulbesuch der Migrantenkinder in Hauptschule, Realschule, Gymnasium auffällig anders: sie stellen eine große Quote in den Hauptschulen und eine geringe in den Gymnasien; ungefähr dreimal so viele Schüler ohne Migrationshintergrund besuchen nach heutigem Stand das Gymnasium, und nur halb so viele eine Hauptschule. Dabei gibt es deutliche Unterschiede zwischen den Nationen [6].

Die Verteilung auf die Schultypen ist nur ein grober Indikator für Chancengleich heit. Genaueres, insbesondere in inhaltlicher Hinsicht, erfährt man über standardisierte Schulleistungstests. Die jüngsten und brauchbarsten Daten stammen aus den genannten PISA-Studien, von denen bisher zwei Messungen (2000 und 2003) ausgewertet worden sind. Sie beziehen sich auf 15-Jährige und die Fächer Mathematik, Naturwissenschaften, Lesen und auf die Problemlösekompetenz.

In den Tests zur Lesekompetenz (PISA 2000) liegen die Migrantenschüler ungefähr 2 Schuljahre hinter den anderen. In Mathematik beträgt ihr Rückstand ebenfalls gut 2 Schuljahre, und in den Naturwissenschaften zwischen 2 und 3 Schuljahren.

Was die alltägliche Sprachpraxis anbetrifft, so ist folgender Befund der jüngsten Studie von Interesse: Wenn man die Migrantenschüler in 3 Gruppen unterteilt, wie oben bereits geschehen - also in Deutschsprachige, Zweisprachige und Fremdsprachige - und die schulischen Leistungen dieser drei Gruppen vergleicht, zeigt sich ein ausgeprägter Zusammenhang zwischen der Alltagssprache und den Lernergebnissen in der Schule, und zwar in dem Sinn, dass der häufigere Gebrauch des Deutschen mit deutlich besseren Schulleistungen einhergeht. Während die „mehrsprachigen“ oder die „fremdsprachigen“ Migranten im Durchschnitt ein bis zwei Schuljahre hinter dem Mittelwert aller Schüler zurück liegen - und zwar nicht nur in der Lesekompetenz, sondern auch in Mathematik -, kommen die „deutschsprachigen" Migranten in vielen Bundesländern mit ihren Schulleistungen nahe an den Gesamt-Durchschnitt heran.

Dies entspricht einer anderen Analyse der Daten aus der ersten PISA-Studie: Hält man die Lesekompetenz konstant - also bei gleicher Lesekompetenz im Deutschen -, gibt es so gut wie keine Unterschiede in den Schulleistungen zwischen den Schülern mit und ohne Migrationshintergrund.

Nach den zitierten Befunden aus diesen methodisch gut gemachten Studien fühlt man sich veranlasst zu sagen: weil die Migrantenschüler schlecht deutsch sprechen, liegen ihre Schulleistungen niedrig. Es ist ja auch plausibel, dass Kinder, die zu Hause und mit ihren Freunden meist russisch, türkisch, griechisch, albanisch, arabisch sprechen, in der ganz auf deutsch funktionierenden Schule Schwierigkeiten haben.

Man darf trotzdem nicht in den Fehler verfallen und aus diesen Daten eine ursächliche Beziehung zwischen den genannten Variablen als erwiesen betrachten. Denn es handelt sich hier um Korrelationen, die sich in Querschnittuntersuchungen ergeben haben, also um das gleichzeitige Auftreten von 2 Variablen in einer Momentaufnahme. Solche Ergebnisse zeigen keine kausalen Beziehungen. Es könnte ja z.B. so sein, dass es sich bei den Schülern mit guten Deutschkenntnissen um besonders intelligente Schüler handelt, die dann natürlich auch gute Schulleistungen erbringen; dann wäre die Intelligenz die Ursache für beides: sowohl für die guten Deutschkenntnisse als auch für die guten Schulleistungen.

Um solchen Problemen aus dem Weg zu gehen, haben die Autoren der genannten Studien versucht, möglichst viele in Frage kommende Variablen zu kontrollieren. Es zeigt sich z. B., dass bei Kontrolle des Sozialstatus der Schüler ein zwar etwas geringerer, 
aber immer noch deutlicher Zusammenhang zwischen der Kompetenz in der L2 und der Schulleistung erhalten bleibt. Das methodische Problem dabei ist: man kann nur diejenigen Variablen kontrollieren, die man auch erhoben hat; andere, die eventuell ebenfalls wirksam sind, bleiben unerkannt.

Darauf weisen im Übrigen die Autoren der genannten Studien selbst immer wieder explizit hin, dass man ihre Daten nicht kausal interpretieren darf. Sie lassen sich dann freilich immer mal wieder hinreißen, plausible korrelative Zusammenhänge kausal zu interpretieren, z. B. mit Formulierungen wie: Die Befunde belegten, „dass die Beherrschung der deutschen Sprache ein wichtiger Bedingungsfaktor für den Kompetenzerwerb" sei. Oder: die Beherrschung der Unterrichtssprache sei „eine entscheidende Voraussetzung für den Lernerfolg in der Schule“ [2].

Wir können also, bis es bessere Daten gibt, einen kausalen Zusammenhang zwischen Kompetenz in der L2 und Schulleistungen als zwar plausibel ansehen, dürfen uns aber nicht in Sicherheit wiegen und davon ausgehen, dass wir eine kausale Beziehung nachgewiesen hätten. Dazu braucht es andere Arten von Forschungsdesigns. Nach diesem Exkurs über forschungsmethodische Probleme bei den berichteten Studien ist es umso erfreulicher, dass vor wenigen Wochen Untersuchungen bekannt oder publiziert worden sind, die zumindest teilweise die für eine Kausalanalyse erforderlichen methodischen Standards einhalten. Einige davon sind Interventionsstudien, in denen Experimental- und Kontrollgruppen miteinander verglichen werden, andere sind Längsschnittuntersuchungen, bei denen man prüfen kann, welche Veränderungen sich über die Zeit bei denselben Personen ergeben und worauf sich diese Veränderungen zurückführen lassen.

I. Eine der Interventionsstudien ist das inzwischen über die Medien bekannt gewordene so genannte Jacobs Sommercamp [7]. Hier hat man 150 Migrantenkinder aus dritten Grundschulklassen im Sommer 2004 in der Nähe von Bremen in Schullandheimen in den Sommerferien einige Wochen betreut und ihnen neben Freizeitaktivitäten ein Theaterspielprogramm sowie systematischen Deutschunterricht (Grammatik) angeboten. Ziel war, ihre Deutschkenntnisse zu verbessern und herauszufinden, auf welche Weise dies am effektivsten geschieht. Der Lernzuwachs wurde bestimmt und mit einer Kontrollgruppe von ca. 80 Schülern verglichen, die ihren normalen Sommer verbrachten. Die Ergebnisse befinden sich noch in der Auswertung, immerhin ist inzwischen so viel deutlich, dass nur der systematische Deutschunterricht eine signifikante und nachhaltige Sprachförderung im Deutschen erbracht hat, nicht aber Theaterspielen oder Freizeitbetreuung; explizite, nicht implizite Sprachförderung hat sich als wirksam erwiesen. Auch in einer kurzen Interventionszeit lassen sich die Deutschkenntnisse von Migrantenschülern signifikant verbessern, sofern man explizit die L2 lehrt; und zwar ohne Förderung der L1.

II. Über einen ungewöhnlich großen und guten Datensatz verfügt eine gerade erst publizierte Längsschnittuntersuchung, bei der man ca. 8.500 Schüler der 9. Klassen im Jahr 2003 untersucht und nach einem Schuljahr wieder getestet hat [8]. Etwa ein Drittel der Schüler ging im Längsschnitt verloren.

Was unsere Frage betrifft, haben sich zwei Hauptbefunde ergeben:

- Zwei- oder Mehrsprachigkeit von Migrantenschülern geht mit guten Leistungsfortschritten in Mathematik einher.

- Schüler, die Zusatzkurse in DaZ (Deutsch als Zweitsprache) besuchen, haben einen besonders geringen Leistungszuwachs in Mathematik.
Das klingt überraschend. Wie lassen sich diese Befunde erklären?

Dass Zweisprachigkeit mit guten Leistungsfortschritten in Mathematik einhergeht, erklärt sich in dieser Studie nicht daraus, dass Mehrsprachigkeit die Lernergebnisse in anderen Bereichen begünstigt. Vielmehr beziehen sich die Längsschnittdaten nur auf die besseren Schüler, nicht auf die ursprünglich in Klasse 9 untersuchten Schüler insgesamt. Denn am Ende der 10. Klasse gab es im Wesentlichen nur noch Gymnasiasten und Realschüler; Hauptschüler, aber auch Sitzenbleiber waren dagegen in der 2. Messung nicht mehr vertreten. Damit war das gesamte schwächere Drittel der Schüler aus der Untersuchung ausgeschlossen. Mit den Worten des Autors: „In der Messwiederholungsstudie können also nur Aussagen über die kompetenzstärkeren Jugendlichen mit Migrationshintergrund gemacht werden." Es ist also die höhere allgemeine kognitive Leistungsfähigkeit, die beides ermöglicht hat: Zweisprachigkeit im Alltag und gute Lernfortschritte in Mathematik.

Entsprechend erklärt sich der ebenfalls zunächst überraschende Befund, dass das Belegen von Kursen in Deutsch als Zweitsprache mit geringerem Zuwachs der Mathematikleistungen kovariiert. Auch hier handelt es sich nicht darum, dass DaZ-Kurse (Deutsch als Zweitsprache) das Mathematiklernen behindern, sondern der Befund beschreibt eine besondere Gruppe, nämlich generell leistungsschwache Migrantenschüler, die deswegen zusätzliche Sprachförderung benötigen und außerdem schwache Leistungen in Mathematik erbringen.

Wir können aus der Studie lernen, dass Bilingualismus für leicht lernende Schüler, die es ohnehin nicht schwer haben mit der Schule, keine Leistungsnachteile mit sich zu bringen scheint. Im Übrigen gelten, bis wir mehr wissen, die Befunde aus den zitierten Surveys, nämlich dass Migrantenschüler nur dann gleiche Schulleistungen erbringen wie Schüler ohne Migrationshintergrund, wenn ihr Deutsch gut ist.

Die zuletzt genannten Studien sind wichtige Schritte auf dem Weg zur Identifikation von Ursachen und Wirkungen. Sie zeigen zwar noch nicht abschließend, aber doch deutlicher, als wir es bisher wissen konnten, was unter normalen schulischen Bedingungen getan werden sollte, wenn man die Schulleistungen dieser Schüler verbessern will.

Bis es genügend belastbare, spezifische Forschungsbefunde gibt, können wir außerdem auf Bestände und Befunde einschlägiger psychologischer Forschungen zurückgreifen, die seit vielen Jahrzehnten betrieben werden und eine große Zahl gesicherter Ergebnisse erbracht haben. Insbesondere ist hier an Erkenntnisse aus der Lernpsychologie zu denken.

Aus Platzgründen lässt sich das hier nicht im Detail darstellen, insbesondere ist aber auf eine Variable hinzuweisen, die sich durchgängig als entscheidend für Lernerfolg (welcher Art auch immer) erwiesen hat: Time on Task [9]. Je mehr Zeit ein Mensch, gleich welchen Alters und gleich bei welcher Aufgabe, mit dem Erlernen einer gestellten Aufgabe verbringt, desto besser sind seine Lernergebnisse - vorausgesetzt, er verwendet diese Zeit auch ganz auf die Lernaufgabe.

Dies scheint ein sehr simples Prinzip zu sein. Es ist allerdings immer wieder z.B. durch die Hoffnung ersetzt worden, dass es eine Übertragung dessen, was man in einem Bereich gelernt hat, auf einen anderen Bereich gebe. Diese Hoffnungen auf Transfer haben sich nur sehr selten erfüllt [10]. Als Beispiel dafür kann der oben diskutierte, vermutete Transfer der Kompetenz in der L1 auf die L2 dienen, für die sich bisher keine belastbaren Belege beibringen ließen. 
Angewandt auf unseren Fall heißt dies: je mehr Zeit Migrantenschüler auf das aktive Erlernen der L2 verwenden, desto höher werden sich ihre Kompetenzen darin entwickeln. Wenn sie dagegen ihre - ja immer begrenzte! - Zeit in das Erlernen der L1 investieren, bleibt naturgemäß weniger für anderes übrig.

Eine Sprache zu erlernen erfordert im Übrigen viel Zeit, wie wir aus unterschiedlichen Studien wissen; man muss die Lerndauer in Jahren ansetzen. Wenn man gute Leistungen in den Schulfächern erzielen möchte, wird es daher eine große Hilfe sein, sich beim Lernen der Verkehrssprache zunächst auf die Schulsprache zu beschränken; das reduziert die Lernaufgabe beträchtlich. Bei der Schulsprache handelt es sich zum einen um die Umgangssprache in schulischen Angelegenheiten, zum anderen um die jeweiligen Sprachen der Schulfächer.

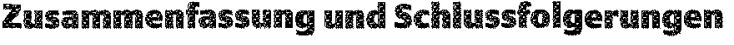

Nach dem heutigen Stand der Forschung lassen sich, holzschnittartig, die vorgetragenen Überlegungen folgendermaßen zusammenfassen und anwenden:

Es gibt eine steigende Anzahl (und eine steigende Quote) von Migrantenschülern. Mittelfristig muss man im Durchschnitt etwa von einem Drittel aller Schüler ausgehen. Es handelt sich also um eine dauerhafte Aufgabe der Schule, diese Kinder optimal zu fördern.

Die Migrantenschüler stammen aus sehr vielen Ländern und sprechen noch mehr Sprachen. Wir haben im Schulalltag eine multilinguale Situation vor uns, keine bilinguale. Auch gibt es eine große regionale Varianz. Die wichtigste Folgerung heißt: Wir sind im Kontext der deutschen Schule auf eine Lingua franca angewiesen. Selbst wenn man von der Richtigkeit bilingualen Unterrichts für alle überzeugt wäre, für fast 200 Herkunftssprachen ist ein solcher kaum realisierbar.

Nach wie vor weisen Migrantenschüler erheblich niedrigere Schulleistungen auf als Schüler ohne Migrationshintergrund. Dies gilt zwar international, in besonders prägnanter Weise aber für Deutschland. Bei uns liegen diese in der Sekundarstufe I um 2-3 Schuljahre zurück. Da abgebrochene Schullaufbahnen, und schlechte Schulleistungen überhaupt, sich für die Betroffenen lebenslang negativ auswirken, müssen erhebliche Anstrengungen unternommen werden, damit diese Schüler begabungsgerechte Schulleistungen erzielen.

Die Schulleistungen der Migrantenschüler kovariieren mit der Beherrschung der Verkehrssprache Deutsch: Defizite in der L2 gehen einher mit schlechten Schulleistungen, Beherrschung der L2 geht einher mit normalen Schulleistungen. Bei aller Vorsicht gegenüber einer kausalen Zuschreibung ist es - nach den Surveydaten (PISA etc.) und aufgrund der Befunde der Lernpsychologie (Time on Task) - hoch plausibel, dass dem Erlernen der L2 absolute Priorität gegeben werden sollte, damit diese Schüler möglichst schnell dem Unterricht ohne Einschränkung folgen können.

Systematische, explizite Förderung der L2 führt zu greifbaren Fortschritten; von impliziter Förderung (Theaterspielen) ist weniger zu erwarten (Jacobs Sommercamp Interventionsstudie). Wir wissen jetzt, dass intensiver Unterricht in Deutsch als zweitsprache erfolgreich sein kann, und zwar ohne Rückgriff auf die L1.

Aus der gerade erschienenen Längsschnittuntersuchung schließlich wissen wir, dass Zweisprachigkeit für begabte Migrantenschüler kein Handicap hinsichtlich ihrer Schulleistungen zu sein scheint; für begabte Kinder ist Zweisprachigkeit zumutbar. Für durchschnittliche und langsame Lerner dagegen ist die Förderung der L1 mit hoher Wahrscheinlichkeit kontraproduktiv, weil sie wichtige Lernzeit in Anspruch nimmt, hier ist die intensive Förderung der Verkehrssprache vorrangig. Der Erkenntnisfortschritt aus diesen Befunden heißt: Man muss das schulische Angebot differenzieren. Guten Schülern kann man auch die L1 anbieten. Allerdings haben auch sie nur eine endliche Lernzeit zur Verfügung, und auch für sie gilt das Gesetz der Time on Task. Auch begabten Schülern fällt eine zweite Sprache nicht einfach zu, sie kostet erhebliche Lernzeit.

Ein Dilemma besteht hinsichtlich der Rolle der Herkunftssprachen. Wir wissen um ihre große Bedeutung für den Einzelnen, auch jenseits von Schulleistungen. Und sie sind ein enormer Reichtum für die Gesellschaft insgesamt. Über eine angemessene Rolle und Förderung der Herkunftssprachen sollte differenziert und öffentlich nachgedacht werden. Was die Schule betrifft, ist es sicherlich das Beste, eine Förderung der L1 anzubieten, sobald die L2 hinreichend beherrscht wird. Ein großes Problem dabei ist die Vielzahl der Sprachen, die wohl kaum ohne computer-unterstützten Unterricht bewältigt werden kann.

Es gibt Grenzfälle, die gesonderter Überlegung bedürfen, z.B. Neuankömmlinge oder Schulanfänger ohne Deutschkenntnisse. Besonders für sie ist es wichtig, das Sprachlernen zunächst auf die Schulsprache zu begrenzen. Im Übrigen bedarf es weiterer Differenzierungen, je nach vorliegenden Bedürfnissen. Geeignet dürfte auch hier der computer-unterstützte Unterricht sein, der sich auch wegen der Streuung der Migrantenschüler übers Land und wegen der geringen Ghettobildung empfiehlt.

Auf jeden Fall brauchen wir verstärkte Forschung, die methodisch so angelegt ist, dass man aus den Resultaten Ursache-Wirkungs-Zusammenhänge ableiten kann. Erst in allerletzter Zeit hat es hier ermutigende erste Schritte gegeben. Inhaltlich muss sich Forschung auch darauf richten, die Effektivität unterschiedlicher einsprachiger Förderprogramme in der Verkehrssprache zu überprüfen.

\section{Pragen zar Selbsticontrolle}

$\nabla$

11. Welche Sprache wird in Deutschland von Migrantenkindern im Alltag am häufigsten benutzt?

a) Die meisten Migrantenkinder sprechen im Alltag häufiger Deutsch als ihre Familiensprache

b) Die meisten Migrantenkinder sprechen im Alltag Deutsch und ihre Familiensprache etwa gleich häufig

c) Die meisten Migrantenkinder sprechen im Alltag häufiger ihre Familiensprache als Deutsch

12. In welcher Sprache sollte Migrantenkinder in der Schule unterrichtet werden?

a) Wenn möglich, sollten der Unterricht in den ersten Klassen in der Muttersprache erfolgen, da perfekte Fähigkeiten in der Muttersprache Voraussetzung für den gelungenen Erwerb einer Zweitsprache sind

b) Bilingualer Unterricht erbringt nachweislich die besten Ergebnisse hinsichtlich der Fähigkeiten in der Muttersprache und in Deutsch

c) Der Unterricht sollte in Deutsch erfolgen, da die Beherrschung der Verkehrssprache über die sozialen Entwicklungschancen eines Kindes entscheidet 
d) Der Unterricht sollte differenzieren: Migranten-Kinder mit hoher Lernfähigkeit können Zweisprachigkeit bewähltigen; lernlangsamere sollten sich auf die Verkehrsprache konzentrieren.

13. Welche Art der Sprachförderung in Deutsch ist für Schüler mit Migrationshintergrund effektiv?

a) Am effektivsten ist eine implizite Sprachförderung z.B. durch Theaterspiele

b) Am effektivsten ist ein systematischer Deutschunterricht mit einer strukturierten Vermittlung u.a. von Grammatikkenntnissen

c) Bislang gibt es keine empirischen Belege für die Überlegenheit der einen oder anderen Vorgehensweise

\section{Literatur}

1 Prenzel M, Baumert J et al. (Hrsg). PISA 2003. Der zweite Vergleich der Länder in Deutschland - Was wissen und können Jugendliche? Münster/New York/München/Berlin. Waxmann 2005

2 Ramm $G$, Walter $O$, Heidemeier $H$, Prenzel $M$. Soziokulturelle Herkunft und Migration im Ländervergleich. In: Prenzel M, Baumert J et al. (Hrsg). PISA 2003. Der zweite Vergleich der Länder in Deutschland - Was wissen und können Jugendliche? Münster/New York/München/ Berlin: Waxmann 2005; 269-298

3 Cummins J. Linguistic interdependence and the educational development of bilingual children. Review of Educational Research 1979; 49: 222-251

4 Hopf $D$. Zweisprachigkeit und Schulleistung bei Migrantenkindern. Zeitschrift für Pädagogik 2005; 51: 236-251 (mit weiterführender Literatur)

5 Limbird C, Stanat P. Sprachförderung bei Schülerinnen und Schülern mit Migrationshintergrund: Ansätze und ihre Wirksamkeit. In: Baumert J, Stanat P, Watermann R (Hrsg). Herkunftsbedingte Disparitäten im Bildungswesen: Differenzielle Bildungsprozesse und Probleme der Verteilungsgerechtigkeit. Wiesbaden: VS Verlag für Sozialwissenschaften 2006; 257-307

6 Baumert J, Schümer G. Familiäre Lebensverhältnisse, Bildungsbeteiligung und Kompetenzerwerb im nationalen Vergleich. In: Baumert J, Artelt C et al. (Hrsg). PISA 2000 - Die Länder der Bundesrepublik Deutschland im Vergleich. Opladen: Leske+Budrich 2002; 159-202

7 Stanat P, Baumert J, Müller AG. Förderung von deutschen Sprachkompetenzen bei Kindern aus zugewanderten und sozial benachteiligten Familien. Evaluationskonzeption für das Jacobs-Sommercamp Projekt. Zeitschrift für Pädagogik 2005; 51: 856-875

8 Walter 0 . Die Entwicklung der mathematischen und der naturwissenschaftlichen Kompetenzen von Jugendlichen mit Migrationshintergrund im Verlauf eines Schuljahres. In: Prenzel M et al. (Hrsg). PISA 2003. Untersuchungen zur Kompetenzentwicklung im Verlauf eines Schuljahres. Münster/New York/München/Berlin. Waxmann 2006 249-275

9 Carroll JB. A model of school learning. Teachers College Record 1963; 64: 723-733

10 Helmke A, Weinert FE. Bedingungsfaktoren schulischer Leistung. In: Weinert FE (Hrsg). Psychologie des Unterrichts und der Schule. Enzyklopädie der Psychologie, Serie l, Pädagogische Psychologie, Band 3. Göttingen 1997; 71-176 